\title{
GLI STUDI DI ALDO PERRONCITO SULLA RIGENERAZIONE DEL NERVO PERIFERICO
}

\author{
VALENTINA CANI (*) \\ Nota presentata dal m.e. Paolo Mazzarello \\ (Adunanza del 7 maggio 2015)
}

\begin{abstract}
SuNTO. - Le rigenerazioni animali costituirono un partecipato oggetto di indagine nel corso del XVIII secolo in tutta Europa. Lazzaro Spallanzani fu uno dei più attivi indagatori del tema e riuscì a dimostrare le capacità rigenerative di molti invertebrati e anche di alcuni vertebrati. All'inizio del XX secolo nel laboratorio di Patologia Generale dell'Università di Pavia diretto da Camillo Golgi, un giovane ricercatore, e poi neolaureato Aldo Perroncito, conseguì dei risultati straordinari riuscendo per la prima volta a comprendere e a descrivere il processo di rigenerazione dei nervi periferici dopo il taglio sperimentale. Le sue ricerche ebbero un grande impatto anche per le successive applicazioni chirurgiche, in effetti i suoi studi aprirono la strada ai primi tentativi di operazione su pazienti feriti al fronte durante la Prima Guerra Mondiale per il ripristino funzionale dei nervi lesi condotti dai medici pavesi Giovanni Verga e Guido Sala.
\end{abstract}

$* * *$

ABSTRACT. - Animal regeneration was a major subject of investigation in the $18^{\text {th }}$ century Europe. Lazzaro Spallanzani was one of the most active experimenters, he was able to demonstrate the regenerative abilities of many invertebrates and even some vertebrates. At the beginning of the $20^{\text {th }}$ century in the General Pathology laboratory of the University of Pavia directed by Camillo Golgi, a young researcher, Aldo Perroncito, succeeded for the first time in understanding and describing the process of regeneration of peripheral nerves after experimental cut. His research had a great impact also for the subsequent surgical applications. Perroncito's studies paved the way for the first attempts at operations on patients wounded during the World War I for the functional restoration of the injured nerves conducted by the University of Pavia graduated medical doctors Giovanni Verga and Guido Sala.

(*) Università degli Studi di Pavia, Italy. E-mail: valentina.cani@unipv.it 


\section{Lo STUdio DELLE RIGENERAZIONI ANIMALI NEL SETTECENTO}

Nel corso del Settecento il fenomeno delle rigenerazioni animali fu oggetto di ampi dibattiti in tutta Europa. Il naturalista francese René Antoine Ferchault de Réaumur notò la capacità dei gamberi di rigenerare le zampe perse e il ginevrino Abraham Trembley scoprì che l'Hydra era in grado di riprodurre non solo porzioni amputate ma anche individui completi "vedi [1], pagg. 52-53". Nella seconda metà del secolo anche Lazzaro Spallanzani - professore e direttore del Museo di Storia Naturale dell'Università di Pavia dal 1769 al 1799 - si dedicò con successo a questo genere di studi. Con una notevole abilità sperimentale riuscì a osservare le capacità rigenerative non solo degli invertebrati ma anche di alcuni vertebrati, ottenendo la riproduzione del capo e della coda nei lombrichi, del capo nelle chiocciole, degli arti e delle mascelle dei tritoni, delle zampe delle giovani rane e dei rospi. Le sue esperienze sulla riproduzione della teste delle lumache, in particolare, fecero grande scalpore sollevando anche interrogativi di ordine filosofico, come il destino dell'anima di questi animali durante gli esperimenti, nei momenti in cui il corpo rimaneva privo della testa "vedi [2], pagg. 54-57; [3]". Nel 1768 Spallanzani pubblicò il Prodromo di un'opera da imprimersi sopra le riproduzioni animali a cui però, nonostante le intenzioni e il grande interesse dei contemporanei, non fece seguito un trattato più organico.

Il successo di tali esperienze fu probabilmente terreno fertile anche per i suoi studenti che muovevano i primi passi nell'osservazione sperimentale. Tra costoro Giuseppe Baronio, laureatosi a Pavia nel 1780, si dedicò alla rigenerazione di parti asportate sperimentalmente spingendosi fino a tentativi di trapianti autoplastici e eteroplastici "vedi [4]". Nell'Ottocento fiorirono gli studi sugli innesti animali, cui si dedicò anche il medico e antropologo Paolo Mantegazza, docente di patologia generale a Pavia tra il 1860 e il 1869 che ebbe tra i suoi allievi anche due astri della biologia cellulare, Giulio Bizzozero e Camillo Golgi. "vedi [5, 6, 7]".

\section{AlCUNi CONTRIBUTI OTTOCENTESCHI ALLO STUDiO DELLA RIGENERAZIONE NELL'UOMO}

I mammiferi hanno una capacità rigenerativa limitata a pochi tessuti. Nell'uomo oltre ai processi di rigenerazione fisiologica si possono verificare eventi rigenerativi in seguito a patologie e traumi a carico di determinati 
organi. Negli anni Ottanta dell'Ottocento, l'Università di Pavia segnò un ulteriore passo in avanti in questo campo di studi grazie alle ricerche compiute da Camillo Golgi che dimostrò la potenzialità rigenerativa del rene osservando il processo di "cariocinesi", da poco teorizzato dal biologo tedesco Walther Flemming, in preparati istologici appartenenti a un paziente colpito da "nefrite parenchimatosa" che aveva attraversato una fase di miglioramento della malattia. Golgi interpretò la nuova formazione di cellule come il segnale di un processo rigenerativo degli elementi epiteliari del parenchima renale "vedi [5] e [8] pagg. 218-219".

Un rilevante contributo teorico giunse qualche anno più tardi dall'Università di Torino grazie agli studi di Giulio Bizzozero, laureatosi appena ventenne a Pavia dove aveva diretto il laboratorio di Patologia Sperimentale frequentato anche da Golgi, con cui strinse un sodalizio scientifico destinato a durare anche dopo la partenza del giovane ricercatore per l'ateneo piemontese. Nel 1894 Bizzozero presentò uno schema di suddivisione dei tessuti in base al loro potenziale generativo e rigenerativo che diventò un elemento fondamentale per la biologia cellulare. Nei tessuti "labili" erano inclusi quelli le cui cellule sono soggette a continue suddivisioni e moltiplicazioni come le cellule del sangue, gli epiteli di rivestimento e alcuni tipi di ghiandole; in quelli "stabili" vi erano quelli con cellule in grado di moltiplicarsi in condizioni fisiologiche solo fino alla nascita, o poco più tardi, come il pancreas, alcune ghiandole, il fegato, il rene la cui generazione era riattivata solo in seguito a processi patologici. Il terzo tipo di tessuti, definiti "perenni", comprendeva quelli con cellule che non erano in grado di riattivarsi nemmeno in seguito a eventi patologici: tra questi rientrava il tessuto nervoso "vedi [5], [9]". Fu a Pavia che un'altra giovane promessa degli studi biologici, Aldo Perroncito, dimostrò per la prima volta il meccanismo di rigenerazione dei tessuti del sistema nervoso periferico.

\section{Aldo Perroncito}

Aldo Perroncito nacque a Torino nel 1882 da una famiglia legata a doppio filo alla scienza. Il padre era Edoardo, parassitologo di fama e docente nella Scuola superiore di veterinaria del capoluogo piemontese, la madre, Erminia Aletti, era cognata di Camillo Golgi e nipote di Giulio Bizzozero. Sembrava quindi quasi scontato che, terminati gli studi, il giovane si iscrivesse alla facoltà di Medicina; nel 1899 si trasferì 
a Pavia dove, fin dal primo anno di studi, frequentò il laboratorio di patologia generale e istologia diretto da Camillo Golgi e si dedicò ben presto allo studio delle terminazioni nervose (Fig. 1). Dopo la laurea, conseguita nel 1901 con una dissertazione dedicata alla rigenerazione dei nervi, divenne assistente nell'Istituto di Patologia Generale, e si dedicò, come molto giovani ricercatori in quegli anni, allo studio dell'apparato reticolare interno, la struttura intracellulare da poco scoperta da Golgi, indagandone i meccanismi citocinetici “vedi [10]”.

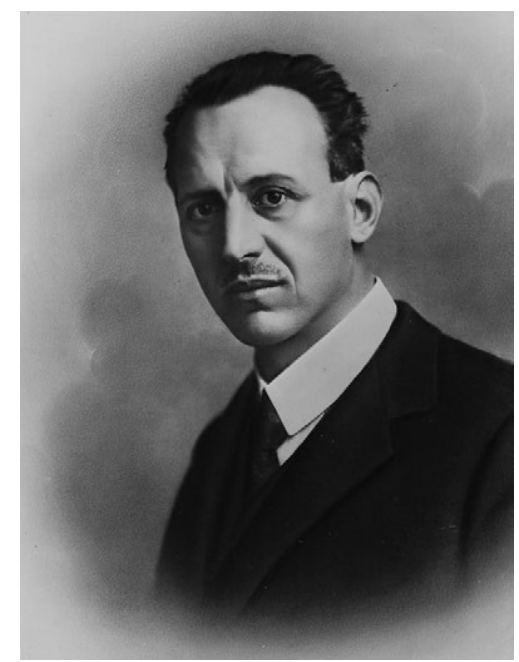

Fig. 1 - Aldo Perroncito, Università di Pavia, Museo per la Storia dell'Università.

Nel 1910 partì per un periodo di perfezionamento all'estero in cui lavorò presso l'Istituto fisiologico di Berlino e in quello di Parassitologia della facoltà medica di Parigi frequentando anche l'Istituto Pasteur per compiere ricerche batteriologiche. Al suo rientro a Pavia fu per qualche anno incaricato dei corsi di Istologia, Patologia Generale, Biologia Generale e Parassitologia e, nel 1915, fu nominato professore straordinario di Patologia Generale a Cagliari, dove ottenne la cattedra nel 1918. In Sardegna si impegnò nella lotta alla malaria, endemica sull'isola, e organizzò una campagna di contrasto alla malattia, cercando di organizzare un sistema organico di profilassi e cura nelle zone minerarie del sud dell'isola a Nebida e Masua "vedi [11, 12, 13]". Il suo impegno nella lotta alla diffusa affezione gli valse una medaglia di bronzo al merito della Sanità Pubblica. 
Durante la guerra prestò servizio in diversi ospedali da campo e si dedicò alla costruzione di laboratori castrensi e stabilimenti di bonifica. Nel 1917 fu ricoverato a Pavia, nell'ospedale militare, per l'aggravarsi dei sintomi di un'infezione tubercolare da cui non si rimise mai completamente e che lo portò a una morte prematura una decina di anni più tardi.

Nel 1922 Perroncito tornò a Pavia come successore di Golgi e divenne preside della facoltà di Medicina nel 1925. Le sue condizioni di salute peggiorarono qualche anno più tardi, sembrò dapprima rimettersi ma le complicanze dovute all'infezione tubercolare mai sopita lo costrinsero a sottoporsi a un intervento chirurgico a Torino; dopo una brevissima convalescenza tornò subito al lavoro, ma il male si aggravò poco tempo dopo e Perroncito morì a Pavia il 21 gennaio 1929 "vedi [14]".

\section{Perroncito e la Rigenerazione DEL NERVO PERIFERICO}

Alla fine dell'Ottocento, nel laboratorio di Patologia Generale, due allievi di Golgi, Giovanni Marenghi e Francesco Purpura, si dedicarono allo studio del meccanismo della rigenerazione dei nervi periferici arrivando a conclusioni contrastanti. Marenghi era convinto che il fenomeno derivasse dallo sviluppo di vie collaterali e non dal ripristino della continuità anatomica del nervo reciso nella cicatrice che si formava dopo il taglio. Purpura invece, impiegando la reazione nera, aveva osservato una continuità anatomica del nervo neoformato, che andava ad attraversare la cicatrice e si portava nel moncone distale. I suoi risultati però erano validi solo per le fibre amieliniche, le uniche in grado di colorarsi, anche se con molta difficoltà, con la reazione nera "vedi [8], pagg. 482-483”.

Perroncito cominciò a dedicarsi allo studio delle terminazioni nervose mentre era ancora studente e ne fece anche il tema della sua dissertazione per la laurea, intitolata La rigenerazione dei nervi, che ottenne con lode nel 1905. Tramite indagini istologiche condotte serialmente riuscì a descrivere per la prima volta in modo esaustivo tutte fasi del processo rigenerativo. Dopo il taglio sperimantale osservò l'emergere, dall'estremità prossimale tagliata, di formazioni di aspetto spiraliforme, poi chiamate anche spirali di Perroncito. Riuscì così a dimostrare che le fibre nervose costituitesi dopo il taglio derivavano da quelle preesistenti, in collegamento con la cellula d'origine "vedi [15-16]". In letteratura sono stati più volte ripresi gli studi condotti sulle rigenerazioni 
da Santiago Ramón y Cajal ma è da attribuire a Perroncito il merito di aver raggiunto per primo conclusioni rilevanti. Fu lo stesso ricercatore spagnolo del resto a riconoscere la priorità del giovane italiano e a proporre che la divisione delle fibre del moncone centrale nelle prime ore dopo il taglio fosse definita fenomeno di Perroncito "vedi [8], pag. 483; [17], pag. 21" (Fig. 2).

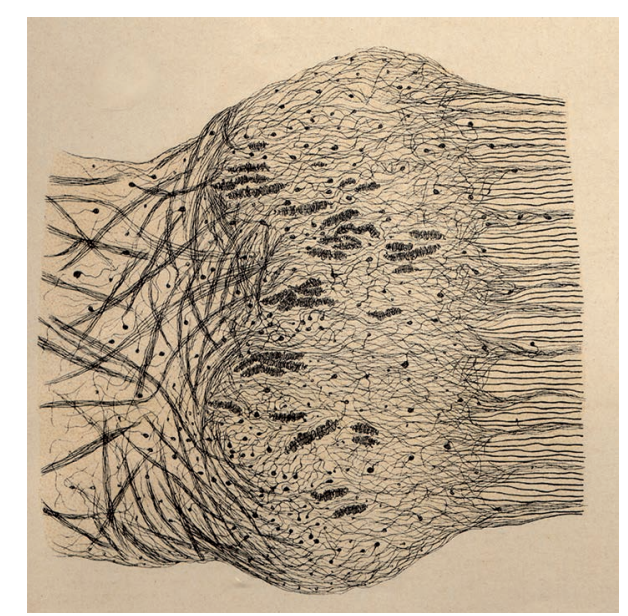

Fig. 2 - Aldo Perroncito, tavola esplicativa delle fasi iniziali del processo di rigenerazione del nervo periferico dopo taglio sperimentale, particolare della tav. 6, in Aldo Perroncito, La rigenerazione dei nervi, Hoepli, Milano, 1908.

Grazie ai suoi fondamentali studi il ricercatore pavese ottenne importanti riconoscimenti internazionali: nel 1907, a venticinque anni, Perroncito ottenne il premio Warren della scuola medica di Boston e nel 1910 il premio Lallemand dell'Académie des Sciences di Parigi.

\section{IL TRATTAMENTO DELLE LESIONI DEL NERVO PERIFERICO}

Nel momento in cui le ricerche di Perroncito iniziarono ad avere risonanza anche in campo internazionale entrò nel laboratorio di patologia generale Giovanni Verga, assistente nell'Istituto di Patologia Chirurgica dimostrativa nell'ospedale San Matteo, deciso a tornare a dedicare del tempo alla ricerca nel laboratorio che aveva già frequentato da studente. Forte anche della sua formazione chirurgica studiò spe- 
rimentalmente la rigenerazione vagliando diverse tecniche in grado di facilitarne il processo, con l'obiettivo di utilizzarle sui pazienti con lesioni dei nervi periferici sui quali non si era mai riusciti a intervenire date le scarse conoscenze sul processo rigenerativo "vedi [18]".

Verga ottenne ottimi risultati nella fase sperimentale in laboratorio e durante la Prima guerra mondiale, insieme al neurologo Guido Sala, operò molti traumatizzati al fronte con lesioni al nervo periferico. I due raccolsero i numerosi interventi in uno studio clinico pionieristico in Italia per la casistica e i risultati riportati che fu comunicato sia alla Società Medico-Chirurgica di Pavia sia all'Istituto Lombardo "vedi $[16,17]$ ".

\section{CONCLUSIONI}

Il rapporto fra guerra e medicina è stato documentato in molti casi particolari. Lo studio dei meccanismi di rigenerazione del nervo periferico avviato da Perroncito ne è un esempio notevole. Lo sviluppo della tecnica chirurgica di riparazione del nervo periferico costituisce inoltre un interessante caso storico di transizione dalla ricerca di base all'applicazione clinica. Nel giro di pochi anni infatti, durante il periodo bellico, le ricerche sperimentali di Aldo Perroncito dimostrarono tutto il loro potenziale terapeutico nel reparto neurologico dell'Ospedale militare del Collegio Borromeo.

\section{BIBLIOGRAFIA}

1. Paolo Mazzarello, Lucio Fregonese (a cura di), Pavia e le svolte della scienza, Libreria CLU, Pavia, 2011.

2. Paolo Mazzarello, Costantinopoli 178: la congiura e la beffa. L'intrigo Spallanzani, Bollati Boringhieri, Torino, 2004.

3. Maria Teresa Monti, Spallanzani e le rigenerazioni animali. L'inchiesta, la comunicazione, la rete, Olschki, Firenze, 2005.

4. Luigi Belloni, Dalle 'riproduzioni animali' di Lazzaro Spallanzani agli 'innesti animali' di Giuseppe Baronio, in: Lazzaro Spallanzani e la biologia del Settecento. Teorie, esperimenti, istituzioni scientifiche. Atti del Convegno di studi Reggio Emilia, Modena, Scandiano, Pavia 23-27 marzo 1981, Giuseppe Montalenti, Paolo Rossi editori, 189-199, Olschki, Firenze,1982.

5. Paolo Mazzarello et al., La rigenerazione del nervo periferico: il contributo della scuola pavese, Neurological Sciences, 2004: 25: 423-425. 
6. Carla Garbarino, Paolo Mazzarello, A strange horn between Paolo Mantegazza and Charles Darwin, Endeavour, 2013: 37(3): 184-187.

7. Maria Carla Garbarino, Paolo Mazzarello, Uno strano trapianto tra Darwin e Mantegazza, Istituto Lombardo (Rend. Scienze), 2013: 147, pp. 127-137.

8. Paolo Mazzarello, Il Nobel dimenticato, Bollati Boringhieri, Torino, 2019.

9. Paolo Mazzarello, Alessandro L. Calligaro, Alberto Calligaro, Giulio Bizzozero: a pioneer of cell biology, Nature Reviews Molecular Cell Biology, 2001: 2(10), pp. 776-81.

10. Aldo Perroncito, Condriosomi, cromidii e apparato reticolare interno nelle cellule spermatiche. Nota preventiva, Rendiconti del R. Istituto lombardo di scienze e lettere, 2018: s. 2: XLI, pp. 988-991.

11. Aldo Perroncito, Esperimento di bonifica umana in località intensamente malarica, Rendiconti del R. Istituto lombardo di scienze e lettere, 1923: LVI, s. 2, pp. 787-792.

12. Aldo Perroncito, Esperimento di bonifica umana in località intensamente malarica. Nota II, Rendiconti del R. Istituto lombardo di scienze e lettere, 1924: LVII, pp. 616-618.

13. Eugenia Tognotti, Per una storia della malaria in Italia. Il caso della Sardegna. Franco Angeli, Milano, 2008.

14. Valentina Cani, Antonella Berzero, Perroncito Aldo, in Dizionario Biografico degli Italiani, vol. 82, Istituto della Enciclopedia Italiana, Roma, 2015, pp. 442-444.

15. Aldo Perroncito, La rigenerazione delle fibre nervose, Archivio per le scienze mediche, 1905: XXIX, pp. 597-606.

16. Aldo Perroncito, La rigenerazione delle fibre nervose. III nota preventiva, Archivio per le scienze mediche, 1906: XXX, pp. 452-462.

17. Javier De Felipe, Edward G. Jones, Cajal's Degeneration and Regeneration of the Nervous System, Oxford University Press, New York, 1991.

18. Paolo Mazzarello, José Maria Pascual, Giovanni Verga, Un Pioniere della Chirurgia del Nervo Periferico, in: Divulgatori di conoscenza, di idee e di metodi. I docenti dell'Università di Pavia raccontati attraverso le loro carte, a cura di Fabio Zucca, Alessandra Baretta, Maria Piera Milani, Edizioni Unicopli, Milano, 2014, pp. 57-79.

19. Guido Sala, Giovanni Verga, Le lesioni dei nervi periferici per ferite d'arma da fuoco (parte prima), Memorie dell'Istituto Lombardo di Scienze e Lettere (Classe di Scienze Matematiche e Naturali), 1916: 21, pp. 337-400; Idem, Le lesioni dei nervi periferici per ferite d'arma da fuoco (parte seconda).

20. Guido Sala, Giovanni Verga, Le lesioni dei nervi periferici per ferite d'arma da fuoco (parte seconda), Memorie dell'Istituto Lombardo di Scienze e Lettere (Classe di Scienze Matematiche e Naturali), 1917: 22, pp. 17-157. 